\title{
Effect of pillow height on the biomechanics of the head-neck complex: Investigation of the cranio-cervical pressure and cervical spine alignment
}

\author{
Sicong Ren ${ }^{1}$, Duo Wai-Chi Wong ${ }^{1,2}$, Hui Yang ${ }^{3}$, Yan Zhou ${ }^{3}$, Jin Lin ${ }^{3}$, Ming Zhang ${ }^{\text {Corresp. }{ }^{1,2}}$ \\ 1 Interdisciplinary Division of Biomedical Engineering, The Hong Kong Polytechnic University, Hong Kong, China \\ 2 The Hong Kong Polytechnic University Shenzhen Research Institute, Shenzhen, China \\ 3 Infinitus (China) Company Ltd., China \\ Corresponding Author: Ming Zhang \\ Email address: ming.zhang@polyu.edu.hk
}

Background: While appropriate pillow height is crucial to maintaining the quality of sleep and overall health, there are no universal, evidence-based guidelines for pillow design or selection. We aimed to evaluate the effect of pillow height on cranio-cervical pressure and cervical spine alignment.

Methods: Ten healthy subjects ( 5 males) aged $26 \pm 3.6$ years were recruited. The average height, weight, and neck length were $167 \pm 9.3 \mathrm{~cm}, 59.6 \pm 11.9 \mathrm{~kg}$, and $12.9 \pm 1.2 \mathrm{~cm}$ respectively. The subjects lay on pillows of four different heights ( $\mathrm{H} 0,110 \mathrm{~mm} ; \mathrm{H1}, 130 \mathrm{~mm} ; \mathrm{H2}, 150 \mathrm{~mm}$; and H3, $170 \mathrm{~mm}$ ). The cranio-cervical pressure distribution over the pillow was recorded; the peak and average pressures for each pillow height were compared by one-way ANOVA with repeated measures. Cervical spine alignment was studied using a finite element model constructed based on data from the Visible Human Project. The coordinate of the center of each cervical vertebra were predicted for each pillow height. Three spine alignment parameters (cervical angle, lordosis distance and kyphosis distance) were identified.

Results: The average cranial pressure at pillow height $\mathrm{H} 3$ was approximately $30 \%$ higher than that at $\mathrm{H} 0$, and significantly different from those at $\mathrm{H} 1$ and $\mathrm{H} 2(p<0.05)$. The average cervical pressure at pillow height $\mathrm{H} 0$ was $65 \%$ lower than that at $\mathrm{H} 3$, and significantly different from those at $\mathrm{H} 1$ and $\mathrm{H} 2(p<$ 0.05). The peak cervical pressures at pillow heights $\mathrm{H} 2$ and $\mathrm{H} 3$ were significantly different from that at $\mathrm{H} 0$ $(p<0.05)$. With respect to cervical spine alignment, raising pillow height from $\mathrm{H} 0$ to $\mathrm{H} 3$ caused an increase of $66.4 \%$ and $25.1 \%$ in cervical angle and lordosis distance, respectively, and a reduction of $43.4 \%$ in kyphosis distance.

Discussion: Pillow height elevation significantly increased the average and peak pressures of the cranial and cervical regions, and increased the extension and lordosis of the cervical spine. The cranio-cervical pressures and cervical spine alignment were height-specific, and they were believed to reflect quality of sleep. Our results provide a quantitative and objective evaluation of the effect of pillow height on the biomechanics of the head-neck complex, and have application in pillow design and selection. 
1 Effect of pillow height on the biomechanics of the head-neck complex: Investigation of the 2 cranio-cervical pressure and cervical spine alignment

3

4 Sicong Ren,\#, Duo Wai-Chi Wong,a,,\#, Hui Yang ${ }^{\mathrm{c}}$, Yan Zhou ${ }^{\mathrm{c}}$, Jin Linc, Ming Zhanga,b,*

5

6 a Interdisciplinary Division of Biomedical Engineering, The Hong Kong Polytechnic University,

7 Hong Kong, China

8 b The Hong Kong Polytechnic University Shenzhen Research Institute, Shenzhen, China

$9 \quad$ c Infinitus (China) Company Limited, China

10 \# These authors contributed equally to this work

$13 *$ Corresponding author:

14 Prof. Zhang Ming

15 Interdisciplinary Division of Biomedical Engineering, The Hong Kong Polytechnic University,

16 Hung Hom, Kowloon, Hong Kong, China

17 Tel: (852) 2766-4939

18 Email: ming.zhang@polyu.edu.hk 


\section{Abstract}

Background: While appropriate pillow height is crucial to maintaining the quality of sleep and overall health, there are no universal, evidence-based guidelines for pillow design or selection. We aimed to evaluate the effect of pillow height on cranio-cervical pressure and cervical spine 23 alignment.

Methods: Ten healthy subjects (5 males) aged $26 \pm 3.6$ years were recruited. The average height, weight, and neck length were $167 \pm 9.3 \mathrm{~cm}, 59.6 \pm 11.9 \mathrm{~kg}$, and $12.9 \pm 1.2 \mathrm{~cm}$ respectively. The subjects lay on pillows of four different heights $(\mathrm{H} 0,110 \mathrm{~mm} ; \mathrm{H} 1,130 \mathrm{~mm} ; \mathrm{H} 2,150 \mathrm{~mm}$; and $\mathrm{H} 3,170 \mathrm{~mm}$ ). The cranio-cervical pressure distribution over the pillow was recorded; the peak and average pressures for each pillow height were compared by one-way ANOVA with repeated measures. Cervical spine alignment was studied using a finite element model constructed based on data from the Visible Human Project. The coordinate of the center of each cervical vertebra were predicted for each pillow height. Three spine alignment parameters (cervical angle, lordosis distance and kyphosis distance) were identified.

Results: The average cranial pressure at pillow height $\mathrm{H} 3$ was approximately $30 \%$ higher than that at H0, and significantly different from those at H1 and $\mathrm{H} 2(p<0.05)$. The average cervical pressure at pillow height $\mathrm{H} 0$ was $65 \%$ lower than that at $\mathrm{H} 3$, and significantly different from those at $\mathrm{H} 1$ and $\mathrm{H} 2(p<0.05)$. The peak cervical pressures at pillow heights $\mathrm{H} 2$ and $\mathrm{H} 3$ were significantly different from that at H0 $(p<0.05)$. With respect to cervical spine alignment, raising pillow height from $\mathrm{H} 0$ to $\mathrm{H} 3$ caused an increase of $66.4 \%$ and $25.1 \%$ in cervical angle and lordosis distance, respectively, and a reduction of $43.4 \%$ in kyphosis distance. 
40 Discussion: Pillow height elevation significantly increased the average and peak pressures of the 41 cranial and cervical regions, and increased the extension and lordosis of the cervical spine. The 42 cranio-cervical pressures and cervical spine alignment were height-specific, and they were 43 believed to reflect quality of sleep. Our results provide a quantitative and objective evaluation of 44 the effect of pillow height on the biomechanics of the head-neck complex, and have application 45 in pillow design and selection. 


\section{$48 \quad$ 1. Introduction}

49 It is estimated that neck pain occurs in nearly a quarter of the population, and poor pillow 50 designs represent important factors contributing to this extremely high incidence (Chiu \& Leung 51 2006; Hoy et al. 2010). Improper pillow support has adverse effects on the cervical spine,

52 leading to neck pain and cervicogenic headache, which ultimately results in poor quality of sleep

53 (Gordon et al. 2011; Ohman 2013).

54 The cervical support pillow, characterized by a B-curve shape, has been proposed as a solution

55 for better support of the cervical spine owing to the pillow prominence under the neck region 56 (Her et al. 2014). It was claimed that this type of cervical pillow design can maintain the spine in 57 a neutral posture and allows the joints and muscles to achieve optimal resting states (Bernateck et al. 2008; Hannon 1999; Her et al. 2014). However, evidence regarding the benefits of cervical pillows was controversial and insubstantial (Shields et al. 2006). Some studies demonstrated that

60 the cervical pillow can treat neck pain and should be regarded as a complement to physical therapy (Bernateck et al. 2008; Erfanian et al. 1998; Persson 2006). On the other hand, it was

62 found that the cervical pillow induced hyper-extension of the neck and was poorly tolerated 63 (Lavin et al. 1997). The review conducted by Shields et al. (2006) criticized the quality of the 64 studies related to cervical pillows and their insufficient statistical power.

65 Pillow height affects comfort and sleeping quality, and was identified as one of the critical 66 factors influencing spinal alignment (Her et al. 2014; Verhaert et al. 2011). However, selecting

67 the correct pillow height can be difficult. Optimal pillow height does not correlate with 68 individual anthropometrical dimensions, such as length or width of the head-neck complex 69 (Erfanian et al. 2004; Wang et al. 2014). People may often choose a pillow based on their 
70 immediate perception and comfort, which could be misleading and may lead to choosing an 71 inappropriate pillow size that induces or worsens neck pain (Erfanian et al. 2004; Leilnahari et al. 72 2011; Liu et al. 2011). The reliability of the rating obtained using questionnaires or feedback is 73 disputable. Users generally rank higher comfort for softer pillows (Hurwitz et al. 2009), though

the perception of comfort may change after a period of adaptation (Gordon et al. 2011). In fact, a firm pillow that may initially seem less comfortable is helpful to stabilize the spine and reduce undesirable spinal distortion (Sacco et al. 2015; Verhaert et al. 2011).

Although there are numerous pillow designs available on the market, a significant number of these are protected by commercial patents, whilst developing designs based on biomechanical research and objective evaluative methods represents an expensive alternative. The present study was motivated by the industry need to evaluate the biomechanical performance of different pillow design elements, with a particular focus on pillow height. Therefore, the objective of the present study was to evaluate the influence of pillow heights on the biomechanics of the headneck complex. It was hypothesized that pillow height would alter the cervico-cranial pressure and alignment. To this end, cervio-cranial pressure was measured as an indicator of weight distribution and comfort (Rithalia \& Gonsalkorale 2000). In addition, the cervical spine alignment was postulated by a simplified finite element model, a simulation-based approach able to reveal the internal features of the spine otherwise difficult to be examined by in vivo experiments and non-invasive means (Wang et al. 2016a). Finally, to validate the computational model, the predicted cranial height was compared with photograph-measured cranial height. 


\section{2. Materials and Methods}

\section{$93 \quad 2.1$ Physical Experiment}

94 2.1.1 Subjects

95 Ten healthy subjects ( 5 male, 5 female), with a mean age of $26 \pm 3.6$ years were recruited at the 96 university campus. The necessary sample size was estimated using large effect size, a test of 97 medium power, and 5\% significance level. The subjects reported no history of chronic 98 myofascial pain, acute injury, or inflection over the spine. The average height, weight, and neck 99 length of the patients were $167 \pm 9.3 \mathrm{~cm}, 59.6 \pm 11.9 \mathrm{~kg}$ and $12.9 \pm 1.2 \mathrm{~cm}$, respectively. The neck 100 length was the distance between the inion and the spinous process of $\mathrm{C} 7$, which was measured in 101 upright standing posture. The study was approved by the Human Subjects Ethics Sub-committee 102 of The Hong Kong Polytechnic University (reference number: HSEARS20140415002). Each 103 participant signed an informed consent form prior to the start of the experiment.

\subsubsection{Material}

105

106

107

108

109

110

111

112

A typical B-shaped cervical pillow (Benelife, Infinitus Co. Ltd., China) made of polyurethane foam was employed. Three polyester elevation mats that were bundled with the cervical pillow were used to heighten the pillow in the experiment. The design and dimensions of the pillow and mats are illustrated in Figure 1.

\section{Figure 1. Design and dimensions of the pillow (a) and elevation mats (b)}

\subsubsection{Equipment}

A pressure sensitive mat (BodiTrak BT1526, Vista Medical Ltd., Winnipeg, MB, Canada) was utilized to measure the pressure distribution on the pillow. The mat consists of 1024 sensors 
113 covering an area of $46.5 \mathrm{~cm}$ by $46.5 \mathrm{~cm}\left(2162.25 \mathrm{~cm}^{2}\right)$. It could provide pressure data with a

114 resolution of $32 \times 32$ points $\left(2.1\right.$ sensors per $\left.\mathrm{cm}^{2}\right)$, and for a maximum pressure value of 26.66

$115 \mathrm{kPa}$. Data were recorded at $50 \mathrm{~Hz}$. The pressure distributions were measured using the FSA 4.1

116 software (BodiTrak, Vista Medical Ltd., Winnipeg, MB, Canada). The peak pressures at the

117 cranial and cervical regions could be readily identified from the pressure distribution outputted

118 by the software. We then identified the site of lowest pressure between the two high-pressure

119 peaks. We approximated that the division between the cranial and cervical regions was located

120 on that intermediate site of lowest pressure. The average pressures of each region could be

121 calculated and extracted using the software.

122 A digital camera (Lumix DMC-Lx5, Panasonic Corp., Osaka, Japan) was used to measure the

123 cranial height and facilitate the validation of the computational model. For each subject, the

124 digital camera was aligned to the position of the right tragus in the sagittal plane with a focal

125 distance of $1 \mathrm{~m}$. The calibration of the alignment was performed using cardboard grids of known

126 dimension, placed at the level of the right tragus.

\subsubsection{Experimental Procedure}

128

129

130

131

132

133

134

Subjects were required to wear shirts without collars. The subjects lay in a supine position on four pillow height conditions assigned as a randomized crossover trial. The four conditions were: without elevator mats $(\mathrm{H} 0)$; with one elevator mat $(\mathrm{H} 1)$; with two elevator mats $(\mathrm{H} 2)$; with three elevator mats (H3). The overall heights for each pillow arrangement were: $110 \mathrm{~mm}$ for H0, 130 $\mathrm{mm}$ for $\mathrm{H} 1,150 \mathrm{~mm}$ for $\mathrm{H} 2$, and $170 \mathrm{~mm}$ for $\mathrm{H} 3$.

For consistency of the results, the pressure sensitive mat was aligned with the top and left borders of the pillow. The subjects were instructed, and were further assisted in order to place the 
135 external occipital protuberance of the skull on a reference point of the mat. The position was

136 such that the neck was placed on the highest point of the cervical pillow. The subjects lay on the

137 mattress in supine position with eyes closed, legs slightly separated, and arms at the sides of the

138 body. Since our pilot test showed that the subjects would adjust their positions, and that the

139 pressure distribution would become steady within 30 seconds, we waited for 30 seconds to begin

140 the measurements for all pillow height conditions. A previous study discussed that such time was

141 sufficient to perform a biomechanical evaluation of the immediate effect (Normand et al. 2005).

142 2.1.5 Statistical Analysis

143 An independent sample T-test was performed to identify anthropometric differences between 144 genders. Normality and sphericity tests were performed, and the pillow height effect was 145 evaluated using ANOVA. Post-hoc adjustment was conducted for pair-wise comparison. All 146 statistical analyses were performed using the statistical software SPSS (SPSS Inc., Chicago, IL, 147 USA).

$148 \quad 2.2$ Computer Simulation (Finite Element Analysis)

149 2.2.1 Model Development

150 The human body model was reconstructed from the dataset acquired from the Visible Human

151 Project that was run by the United States National Library of Medicine (Ackerman 1998). The

152 subject was a 38-year-old male, weighed $69 \mathrm{~kg}$ and was 178-cm tall. Three-dimensional 153 geometries of the head and upper trunk, including the skull, brain, part of the rib cage, scapula, 154 clavicle, cervical vertebrae, intervertebral discs and encapsulated soft tissue, were reconstructed 155 using the Mimics software (Materialise, Leuven, Belgium). The interaction between bones 156 (articular joints) was assumed frictionless. Thereafter, the finite element package, Abaqus 
157 (Dassault Systèmes, RI, USA) was utilized for mesh creation and subsequent analysis. Figure 2

158 shows the finite element model of the human body and of the pillow.

159

160

161

162

163

164

165

166

167

168

169

170

171

172

173

174

175

176

177

Figure 2. Finite element model of the head-neck complex with pillow: (a) model geometry in frontal view; (b) side and cross-section views of the head-neck complex resting at $\mathrm{H3}$ condition.

The geometries of the pillow and elevator mats were digitized using a hand-held 3D scanner (Xbox One Kinect, Microsoft, USA). The interaction between the body and the pillow was assumed frictionless. All geometrical parts were meshed with tetrahedral elements (C3D4). The approximate densities were set to $1700 \mathrm{~kg} / \mathrm{m}^{3}$ for bone and $1000 \mathrm{~kg} / \mathrm{m}^{3}$ for other soft tissues (Guo et al. 2015). The materials of all parts were linearly elastic, except for the encapsulated soft tissue, which was modeled as hyperelastic with a second-order polynomial strain energy potential (Cheung et al. 2005). The material properties of the finite element model are listed in Table 1.

\section{Table 1. Material properties of the finite element model}

\subsubsection{Boundary and Loading Conditions}

First, the body model was placed on a flat base and gravity $\left(9.8 \mathrm{~m} / \mathrm{s}^{2}\right)$ was applied. Next, the pillow model was put under the flat base and gradually risen, making contact with and flexing the head-neck complex until the pillow was resting over the flat base. The predicted displacement of the vertebral column was presented based on the coordinates of each cervical vertebra center. That displacement was then used in order to assess the cervical spine alignment under the four pillow conditions. 
178

179

180

181

182

183

184

185

186

187

188

189

190

191

192

193

194

195

196

197

198

\subsubsection{Output and Parameters}

The coordinates of the centers of the seven cervical vertebrae predicted by the model were connected in a dot-to-dot fashion to create a cubic spline curve that represented the cervical spine under the four conditions. In Figure 3, we show the three parameters that we used in order to determine the cervical spine alignment in the sagittal plane (Haex 2004; Verhaert et al. 2011). The cervical angle $(\mathrm{CA})$ represents an overall elevation of the head and was defined as the angle between the horizontal axis and the line that connects the first cervical vertebra and the seventh cervical vertebra. The lordotic distance (LD) and the kyphotic distance (KD) represent the regional curvature of the lower and upper cervical spine respectively. The LD and KD were quantified as the maximal distance from the lordotic and kyphotic curves, respectively, to the regression straight line (least square line) of the seven vertebrae.

\section{Figure 3. Illustration of the parameters for cervical spine alignment: $\mathrm{CA}$, cervical angle;}

\section{LD, lordosis distance; KD, kyphosis distance. The dashed line is a cubic spline of the} vertebrae center coordinates.

\subsubsection{Validation}

The predicted and measured cervical pressures during supine position under the four pillow conditions were compared. Similarly, the predicted values for cranial height were compared to those measured on photographic images. Cranial height was defined as the vertical distance in the sagittal plane from the bed surface to the right tragus. 


\section{Results}

\subsection{Pre-hoc Test}

202 The influence of gender on the body height, weight and neck was evaluated. Levene's test 203 showed that the variances regarding the anthropometrics were homogeneous, and all 204 anthropometric data were significantly different between male and female subjects $(p<0.05)$, as 205 shown in Table 2.

\section{Table 2 Anthropometric data between male and female subjects}

207

208

209

210

211

212

213

214

215

216

217

218

A mixed ANOVA $(n=5)$ was performed to evaluate the association of gender and pillow height with the values of pressure (Table 3). Mauchly's test of sphericity indicated that the assumption of sphericity of the pressure data was not violated $(p>0.05)$, which was further confirmed using the Shapiro-Wilk normality test $(p>0.05)$. Nevertheless, the interaction effect between gender and pillow height was not significant $(p>0.05)$. Under the premise that pillow designs are seldom marketed as gender-oriented, we decided to pool the data and discard the gender factor.

\section{Table 3 Mixed ANOVA (n=5) on gender and pillow height conditions demonstrating no significant interactions between the factors}

After the data were pooled $(n=10)$, one-way repeated measures ANOVA was performed in order to assess the effect of the different pillow heights on the cranial and cervical pressures. The assumption of sphericity of the data was not violated $(p>0.05)$, but the cervical peak pressures under H0 condition did not pass the Shaprio-Wilk normality test $(p=0.03)$. While it is believed 
219 that ANOVA is relatively robust for normally distributed data, the Friedman test was further

220 performed (i.e. after ANOVA) for the non-normal data in order to verify the result.

\subsection{Cranio-cervical Pressure Measurement}

222 Figure 4 presents the cranio-cervical pressure distribution of a typical subject under the four 223 pillow height conditions. The contact area generally increased with increasing pillow height,

224 while the peak pressure shifted inferiorly from the cranial region to the cervical region. The 225 overall pressure seemed to increase for $\mathrm{H} 3$ condition. from one typical subject

228 The average and peak pressures of the distributions were analyzed, and the mean values of these two parameters for the 10 subjects are shown in Table 2 . There were statistically significant differences among pillow height conditions in terms of average and peak pressures, as determined by one-way repeated measures ANOVA $(p<0.05)$, and the overall effect size $\left(\eta^{2}\right)$ was prominent $\left(\eta^{2}>0.14\right)$. The effect sizes for the average and peak pressures were, respectively: 0.504 and 0.338 for the cranial region; and 0.693 and 0.568 for the cervical region. After the Bonferroni adjustment, the differences regarding average pressures at the cranial and cervical regions were also statistically significant for some of the pair-wise comparisons. The average pressure at the cranial region under $\mathrm{H} 3$ was about 30\% higher than that under H0 $(p<0.0125$,

237 Cohen's $d=1.28$, and significantly different from those under the other conditions (H3 vs. H1: $p<0.0125$, Cohen's $\mathrm{d}=1.12 ; \mathrm{H} 3$ vs. H2: $p<0.0125$, Cohen's $\mathrm{d}=1.11$ ). In addition, the average

239 pressure at the cervical region under H0 was 65\% lower than that under H3 $(p<0.0125$, Cohen's 240 $\mathrm{d}=2.22$ ) and significantly different from those under the other conditions (H0 vs. H1: $p<$ 
2410.0125 , Cohen's $\mathrm{d}=1.2$; H0 vs. H2: $p<0.0125$, Cohen's $\mathrm{d}=2.07$ ). The peak pressures at the

242 cervical region under $\mathrm{H} 2$ and $\mathrm{H} 3$ were significantly different from that under $\mathrm{H} 0$ (respectively: $p$

$243<0.0125$, Cohen's $d=1.27 ; p<0.0125$, Cohen's $\mathrm{d}=1.57)$.

244 Table 4. Mean values $(n=10)$ of the average and peak pressures at the cranial and cervical regions under the four pillow height conditions

246 The assumption of normality was violated for the peak pressure at the cervical region under H0;

247 thus, the Friedman test was performed after ANOVA. The pressure values significantly differ

248 between tests with different pillow heights $(p<0.01$, Kendall's $\mathrm{W}=0.50)$, and the values

249 increased with increased pillow height. Post-hoc analysis using the Wilcoxon signed-rank tests

250 was conducted with a Bonferroni correction. Both $\mathrm{H} 2$ and $\mathrm{H} 3$ were significantly different from

251 H0 (H2 vs. H0: $p<0.0125, \mathrm{r}=0.60$; H3 vs. H0: $p<0.0125, \mathrm{r}=0.60$ ), which was coherent with

252 the ANOVA results (Table 2).

253

254

255

256

257

258

259

260

261

262

\subsection{Predicted Cervical Spine Alignment}

The cervical spine alignment/curvature was predicted using the coordinate centers of the vertebrae as presented in Figure 5. There was an anterior shift (upshift) of the spine as the pillow height increased. The cervical spine alignment parameters for the method illustrated in Figure 3 are listed in Table 3. As the pillow height increased from $\mathrm{H} 0$ to $\mathrm{H} 3, \mathrm{CA}$ increased by $66.4 \%$, KD decreased by $43.4 \%$, and LD increased by $25.1 \%$, indicating an overall extension of the headneck in both the upper and lower cervical regions. The point of transition from the upper cervical kyphosis and lower cervical lordosis was located at the third cervical vertebra.

Figure 5. Finite element predicted position of the cervical vertebrae under the four pillow height conditions 
263 Table 5. Cervical spine alignment parameters in the four pillow height conditions predicted

\subsection{Validation}

266

267

Validation was conducted by comparing the peak cranial and cervical pressures and the cranial height between the finite element prediction and the physical experiment (Figure 6). Both the prediction and the experiment exhibited a consistently increasing trend in pressure as the pillow was elevated. The findings were generally in agreement, given the variance of the experiment.

\section{Figure 6. Comparison between physical experiment and finite element analysis under the} four pillow height conditions: (a) peak cranio-cervical pressure; (b) cranial height

\section{Discussion}

As humans spend a third of their life sleeping, the importance of a high quality of sleep is undeniable. Poor pillow design provides inadequate support of the cervical spine, resulting in discomfort and pain (Liu et al. 2011; Wang et al. 2014). While comfort and perception are commonly evaluated, their validity and correlation with the functional outcome of using a pillow with a particular design remain unclear (Gordon et al. 2011; Her et al. 2014; Hurwitz et al. 2009; Liu et al. 2011). Pillow manufacturers claim various benefits associated with their pillow designs, some of which allow the pillow height to be adjustable; however, such claims often lack scientific support and rigorous verification, and rarely provide guidelines for the selection of a pillow with optimal height when more are available. In this context, the present study aimed to investigate the effect of pillow height on the biomechanics of the head-neck complex, and provide quantitative and objective data to facilitate an evidence-based pillow design and selection process. Interfacial pressure and spinal alignment were selected as the parameters of 
285 interest, as interfacial pressure accounts for tactile comfort, and optimal spinal alignment could

286 allow relax and recovery for muscles and soft tissue during sleeping (Kyung \& Nussbaum 2008;

287 LeBlanc et al. 1994).

288 The difference between the forces supporting the head and those supporting the neck reflects the 289 capability to maintain spine alignment, and has been included in pillow design as a key factor 290 (Huysmans et al. 2006; Verhaert et al. 2011). In our study, elevating the pillow re-positioned the 291 center of supporting force and redistributed the load bearing ratio between the cranial and 292 cervical regions, as shown by the change in pressure distribution (Figure 4) and pressure values 293 (Table 2). Lavin et al. (1997) suggested that distribution of the supporting pressure may be 294 achieved by a water-based pillow, as such a pillow that responds more easily to the contour of 295 the body. In addition, pressure can be considered a measure of comfort (Hänel et al. 1997). 296 Specifically, higher pressure may demonstrate sufficient support but, may be poorly tolerated. 297 This aspect is related to the fact that body contact pressure affects fluid transfusion across soft 298 tissue (Adams \& Hutton 1983). Skin contact pressure below 4.20kPa was believed to allow 299 capillary perfusion and hence better comfort (Bridges et al. 2003). Our findings indicated that the 300 above-mentioned pressure threshold was exceeded, suggesting poor tolerance of certain pillow 301 heights; however, due to the study design, these findings do not take into account the influence 302 of turning during sleep and the consequent limitation on the time that the skin contact pressure is 303 above or below the threshold (time effect). We were thus not able to provide the optimal range 304 for contact pressure and pressure ratio between the cranial and cervical regions that would 305 achieve suitable supporting force as well as comfort. Further investigations into such aspects are 306 warranted. 
307 Cervical spine alignment or curvature represent key factors affecting sleep parameters (Verhaert

308 et al. 2011). In disregard of possible radiation hazard to the users, lateral radiographs have been

309 used for evaluating the influence of pillow material and shape on the spine curvature (Jackson

310 2010; Persson \& Moritz 1998; Wang et al. 2014), with reflective markers or scanners being used

311 to estimate the positions of the vertebrae (Gordon et al. 2011; Verhaert et al. 2011). The recent

312 advance in computer simulation technology has enabled non-invasive and more detailed

313 investigations regarding the biomechanics of pathology, injury, surgery, and body-interface

314 design (Cheung et al. 2009; Wong et al. 2016; Wong et al. 2015; Wong et al. 2014; Yu et al.

315 2013). Finite element analysis provide a versatile platform to examine the internal biomechanical

316 characteristics of the human body in a controlled environment, using pre-defined parameters

317 (Fagan et al. 2002; Wang et al. 2016b). However, few attempts focused on the effect of the

318 pillow or mattress design (Leilnahari et al. 2011), and some simulations employed a largely

319 simplified geometry of the human body (Fagan et al. 2002; Haex 2004). The present study

320 utilized a three-dimensional, anatomically detailed, finite element model of the human body, and

321 pursued a novel exploration regarding spine alignment when using different pillow designs.

322 An overall angulation between the head-neck complex and the sleeping surface is often 323 discussed in the media and in advertisements from pillow manufactures. A CA of $15^{\circ}$ was noted

324 (Takano 2016), but to our knowledge, no further literature can be found in support of this claim.

325 In fact, CA or the head alone is insufficient to control and optimize the head-neck posture, since

326 the biomechanics of the upper and lower cervical spine are regional in nature (Takeshima et al.

327 2002). The upper cervical spine is generally kyphotic, while the lower cervical spine is lordotic;

328 and these aspects cause these regions of the spine to work differently during different motions

329 (Takeshima et al. 2002). Our findings suggested that, although extension was noted in both the 
330 upper and cervical spine, pillow height predominantly affected the alignment or posture of the

331 upper cervical spine, where LD and KD changed by $43 \%$ and $25 \%$, respectively, for an increase

332 of $60 \mathrm{~mm}$ in pillow height (from $\mathrm{H} 0$ to $\mathrm{H} 3$ ). The B-shaped cervical pillow supports the head and

333 the neck separately, which could, in principle, facilitate regional manipulation of the upper and

334 lower cervical alignment to achieve better support for both the head and neck. However, it is

335 difficult to determine the ideal heights for each segment of the cervical pillow such that the

336 muscles and intervertebral discs can relax and recover from the continuous loading they bear

337 throughout the day (LeBlanc et al. 1994).

338 It is important to note that the implications of the present findings are limited by the

339 simplifications and assumptions inherent to the simulation. We assumed that, during sleep, the

340 cervical spine alignment is predominantly accounted for by the bony structure and the

341 encapsulated soft tissue (Haex 2004; Izzo et al. 2013). The internal constitution of the ligaments

342 and tendons was not taken into consideration, which may have resulted in underestimating the

343 stability of the spine. The coefficient of friction between the head and pillow was considered

344 negligible, since we assumed that the layer of hair allows relatively free sliding between the head

345 and the pillow. However, the properties of the layer of hair vary significantly among individuals,

346 as does the preference for pillowcase. We also did not consider the skin-pillow interaction in the

347 exposed regions. Nonetheless, we expect that friction and shear, which were along the tangential

348 direction, should have little effect on the prediction of spine alignment in the sagittal plane. The

349 model was constructed based on the supine position, which is typically employed during clinical

350 image scanning but may not necessarily reflect the strain-free state. The evaluation and

351 recreation of appropriate strain-free conditions remains a challenges in the field of finite element

352 analysis (Panzer \& Cronin 2009). Hence, the simulation was limited to predicting the 
353 displacement and angulation of the vertebrae. Future study should anticipate the internal

354 distribution of strain for different pillow height. There were also limitations regarding the 355 accuracy of the physical experiments; for example, a certain degree of imprecision may have

356 been involved in partitioning the cervical and cranial regions.

357 The applicability of finite element analysis has repeatedly been discredited on account of its 358 single-subject design (Wong et al. 2016). While finite element models can provide deterministic 359 predictions to answer questions based on the structure-function relationship, the generalization of 360 results would often be hindered by the specific set of assumptions and loading cases pertaining to 361 the single-subject model (Walmsley et al. 2013; Wong et al. 2016). In the present study, the data 362 used to build the finite element model were taken from the Visible Human Project (Ackerman 363 1998), which is commonly used and recognized as representative (Elkins et al. 2016; Panzer et al. 364 2012). The age of the model subject was outside the age range of the 10 subjects involved in our 365 experimental investigations. However, it has been suggested that pillow performances are not sensitive to body dimensions and age (Erfanian et al. 2004; Wang et al. 2014); our pre-hoc test 367 also demonstrated that gender had significant effect on body dimension but did not have any association with response to pillow height. Therefore, we consider that the model was representative.

From the industrial point of view, the first goal is to recognize the biomechanical parameter ranges that can achieve optimal sleep comfort and quality, which represents an ongoing and

372 challenging task. The second goal is to learn how to achieve this optimal set of parameters by the 373 complex interactions of various pillow design elements. In this study, the biomechanics of pillow

374 height, a critical design parameter, were described for the supine position, since supine lying has 375 less variation compared to other sleeping postures. Nonetheless, considerations of behavior and 
376 biological context such as sleeping position, duration, and quality represent further challenges

377 ahead.

378 5. Conclusion

379 The present study integrated experimental investigations of cranio-cervical pressure and 380 computational predictions of spinal alignment to assess the influence of pillow heights on the 381 biomechanics of the head-neck complex. There was a statistically significant increase in the peak 382 and average pressure in both the cranial and cervical regions $(p<0.05)$. The computer simulation 383 (finite element analysis) showed that the head-neck complex was elevated and extended upon 384 increasing pillow height. However, the degree of extension differed between the upper and lower 385 cervical spine. Pillow height may represent an important and influential parameter to be 386 considered in terms of the biomechanical performance of specific pillow designs. 
Ackerman MJ. 1998. The visible human project. Proceedings of the IEEE 86:504-511.

Adams M, and Hutton W. 1983. The effect of posture on the fluid content of lumbar intervertebral discs. Spine 8:665-671.

Bernateck M, Karst M, Merkesdal S, Fischer MJ, and Gutenbrunner C. 2008. Sustained effects of comprehensive inpatient rehabilitative treatment and sleeping neck support in patients with chronic cervicobrachialgia: a prospective and randomized clinical trial. International Journal of Rehabilitation Research 31:342-346.

Bridges EJ, Schmelz JO, and Mazer S. 2003. Skin interface pressure on the NATO litter. Military medicine 168:280-286.

Cheung JT-M, Yu J, Wong DW-C, and Zhang M. 2009. Current methods in computer-aided engineering for footwear design. Footwear science 1:31-46.

Cheung JT-M, Zhang M, Leung AK-L, and Fan Y-B. 2005. Three-dimensional finite element analysis of the foot during standing-a material sensitivity study. Journal of Biomechanics 38:1045-1054.

Chiu TT, and Leung AS. 2006. Neck pain in Hong Kong: a telephone survey on prevalence, consequences, and risk groups. Spine 31:E540-E544.

Elkins J, Marsh JL, Lujan T, Peindl R, Kellam J, Anderson DD, and Lack W. 2016. Motion Predicts Clinical Callus Formation. J Bone Joint Surg Am 98:276-284.

Erfanian P, Hagino CC, and Guerriero RC. 1998. A preliminary study assessing adverse effects of a semicustomized cervical pillow on asymptomatic adults. The Journal of the Canadian Chiropractic Association 42:156-162.

Erfanian P, Tenzif S, and Guerriero RC. 2004. Assessing effects of a semi-customized experimental cervical pillow on symptomatic adults with chronic neck pain with and without headache. The Journal of the Canadian Chiropractic Association 48:20-28.

Fagan M, Julian S, and Mohsen A. 2002. Finite element analysis in spine research. Proceedings of the institution of mechanical engineers, part $h$ : journal of engineering in medicine 216:281-298.

Gordon SJ, Grimmer-Somers KA, and Trott PH. 2011. A randomized, comparative trial: does pillow type alter cervico-thoracic spinal posture when side lying? Journal of multidisciplinary healthcare 4:321-327.

Guo L, Zhang M, and Teo E-c. 2015. Spine model for vibration analysis. In: Zhang M, and Fan Y, eds. Computational Biomechanics of the Musculoskeletal System. NW, USA: CRC Press, 175-197.

Haex B. 2004. Back and bed: ergonomic aspects of sleeping: CRC press.

Hänel S-E, Dartman T, and Shishoo R. 1997. Measuring methods for comfort rating of seats and beds. International Journal of Industrial Ergonomics 20:163-172.

Hannon JC. 1999. Pillow talk: the use of props to encourage repose. Journal of Bodywork and Movement Therapies 3:55-64.

Her J-G, Ko D-H, Woo J-H, and Choi Y-E. 2014. Development and Comparative Evaluation of New Shapes of Pillows. Journal of physical therapy science 26:377-380.

Hoy D, Protani M, De R, and Buchbinder R. 2010. The epidemiology of neck pain. Best Practice \& Research Clinical Rheumatology 24:783-792.

Hurwitz EL, Carragee EJ, van der Velde G, Carroll LJ, Nordin M, Guzman J, Peloso PM, Holm LW, Côté P, and Hogg-Johnson S. 2009. Treatment of neck pain: noninvasive interventions: results of the Bone and Joint Decade 2000-2010 Task Force on Neck Pain and Its Associated Disorders. Journal of manipulative and physiological therapeutics 32:S141-S175. 
432

433

434

435

436

437

438

439

440

441

442

443

444

445

446

447

448

449

450

451

452

453

454

455

456

457

458

459

460

461

462

463

464

465

466

467

468

469

470

471

472

473

474

475

476

477

Huysmans T, Haex B, De Wilde T, Van Audekercke R, Vander Sloten J, and Van der Perre G. 2006. A 3D active shape model for the evaluation of the alignment of the spine during sleeping. Gait \& posture 24:54-61.

Izzo R, Guarnieri G, Guglielmi G, and Muto M. 2013. Biomechanics of the spine. Part I: spinal stability. European journal of radiology 82:118-126.

Jackson R. 2010. The Classic: The Cervical Syndrome. Clinical Orthopaedics and Related Research ${ }^{\circledR}$ 468:1739-1745.

Kyung G, and Nussbaum MA. 2008. Driver sitting comfort and discomfort (part II): Relationships with and prediction from interface pressure. International Journal of Industrial Ergonomics 38:526538.

Lavin RA, Pappagallo M, and Kuhlemeier KV. 1997. Cervical pain: a comparison of three pillows. Archives of physical medicine and rehabilitation 78:193-198.

LeBlanc AD, Evans HJ, Schneider VS, Wendt III RE, and Hedrick TD. 1994. Changes in intervertebral disc cross-sectional area with bed rest and space flight. Spine 19:812-817.

Leilnahari K, Fatouraee N, Khodalotfi M, Sadeghein MA, and Kashani YA. 2011. Spine alignment in men during lateral sleep position: experimental study and modeling. Biomed Eng Online 10:103.

Liu S-F, Lee Y-L, and Liang J-C. 2011. Shape design of an optimal comfortable pillow based on the analytic hierarchy process method. Journal of chiropractic medicine 10:229-239.

Normand MC, Descarreaux M, Poulin C, and Richer N. 2005. Biomechanical effects of a lumbar support in a mattress. The Journal of the Canadian Chiropractic Association 49:96.

Ohman A. 2013. A pilot study, a specially designed pillow may prevent developmental plagiocephaly by reducing pressure from the infant head. Health 5:32-37.

Panzer MB, and Cronin DS. 2009. C4-C5 segment finite element model development, validation, and load-sharing investigation. Journal of Biomechanics 42:480-490.

Panzer MB, Myers BS, Capehart BP, and Bass CR. 2012. Development of a finite element model for blast brain injury and the effects of CSF cavitation. Annals of biomedical engineering 40:1530-1544.

Persson L. 2006. Neck pain and pillows-A blinded study of the effect of pillows on non-specific neck pain, headache and sleep. Advances in Physiotherapy 8:122-127.

Persson L, and Moritz U. 1998. Neck support pillows: a comparative study. Journal of manipulative and physiological therapeutics 21:237-240.

Rithalia SV, and Gonsalkorale M. 2000. Quantification of pressure relief using interface pressure and tissue perfusion in alternating pressure air mattresses. Archives of physical medicine and rehabilitation 81:1364-1369.

Sacco IC, Pereira IL, Dinato RC, Silva VC, Friso B, and Viterbo SF. 2015. The Effect of Pillow Height on Muscle Activity of the Neck and Mid-Upper Back and Patient Perception of Comfort. Journal of manipulative and physiological therapeutics 38:375-381.

Shields N, Taylor N, Polak T, and Capper J. 2006. Are cervical pillows effective in reducing neck pain? New Zealand Journal of Physiotherapy 34:3-9.

Takano J. 2016. A Pillow That Can Make You Healthy and Sleep Well. Available at http://www.pyroenergen.com/articles08/healthy-pillow.htm (accessed 31 May 2016).

Takeshima T, Omokawa S, Takaoka T, Araki M, Ueda Y, and Takakura Y. 2002. Sagittal alignment of cervical flexion and extension: lateral radiographic analysis. Spine 27:E348-E355.

Verhaert V, Haex B, Wilde TD, Berckmans D, Verbraecken J, Valck Ed, and Sloten JV. 2011. Ergonomics in bed design: the effect of spinal alignment on sleep parameters. Ergonomics 54:169-178.

Walmsley CW, McCurry MR, Clausen PD, and McHenry CR. 2013. Beware the black box: investigating the sensitivity of FEA simulations to modelling factors in comparative biomechanics. PeerJ 1:e204. 
478

479

480

481

482

483

484

485

486

487

488

489

490

491

492

493

494

495

496

497
Wang J-C, Chan R-C, Wu H-L, and Lai C-J. 2014. Effect of pillow size preference on extensor digitorum communis muscle strength and electromyographic activity during maximal contraction in healthy individuals-a pilot study. Journal of the Chinese Medical Association:182-187.

Wang J, Lui Z, Qian Z, and Ren L. 2016a. Soft tissue artifact evaluation of the cervical spine in motion patterns of flexion and lateral bending: a preliminary study. PeerJ 4:e1893.

Wang Y, Wong DW-C, and Zhang M. 2016b. Computational models of the foot and ankle for pathomechanics and clinical applications: a review. Annals of biomedical engineering 44:213221.

Wong DW-C, Niu W, Wang Y, and Zhang M. 2016. Finite element analysis of foot and ankle impact injury: risk evaluation of calcaneus and talus fracture. PloS one 11:e0154435.

Wong DW-C, Wang Y, Zhang M, and Leung AK-L. 2015. Functional restoration and risk of non-union of the first metatarsocuneiform arthrodesis for hallux valgus: A finite element approach. Journal of Biomechanics 48:3142-3148.

Wong DW-C, Zhang M, Yu J, and Leung AK-L. 2014. Biomechanics of first ray hypermobility: An investigation on joint force during walking using finite element analysis. Medical Engineering \& Physics 36:1388-1393.

Yu J, Cheung JT-M, Wong DW-C, Cong Y, and Zhang M. 2013. Biomechanical simulation of high-heeled shoe donning and walking. Journal of Biomechanics 46:2067-2074. 
Figure 1

Design and dimensions of pillow and elevation mats

Figure 1. Design and dimensions of the pillow (a) and elevation mats (b)

(a)

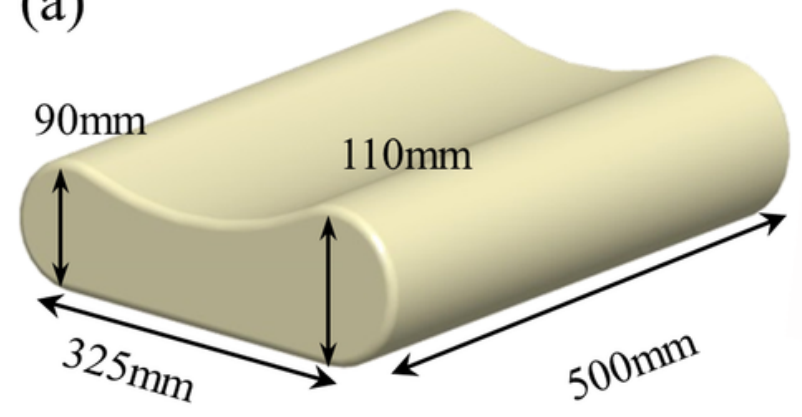

(b)

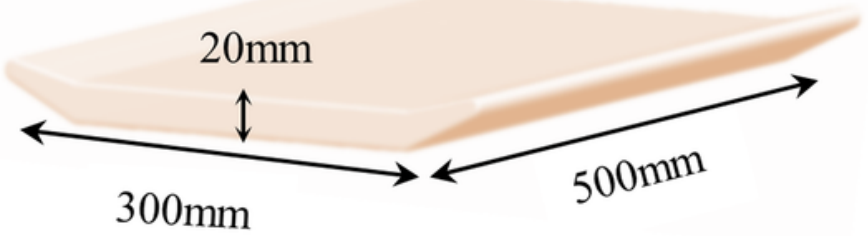




\section{Figure 2}

Finite element model of the head-neck complex with pillow

Figure 2. Finite element model of the head-neck complex with pillow: (a) model geometry in frontal view; (b) side and cross-section view of the head-neck complex resting at $\mathrm{H} 3$ condition.

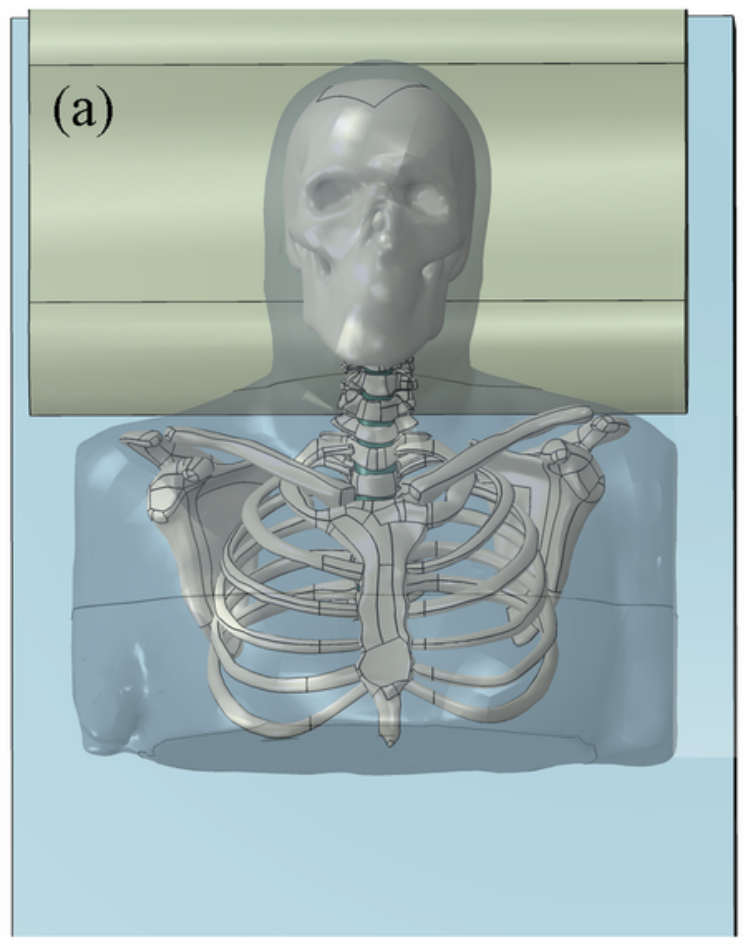

(b)

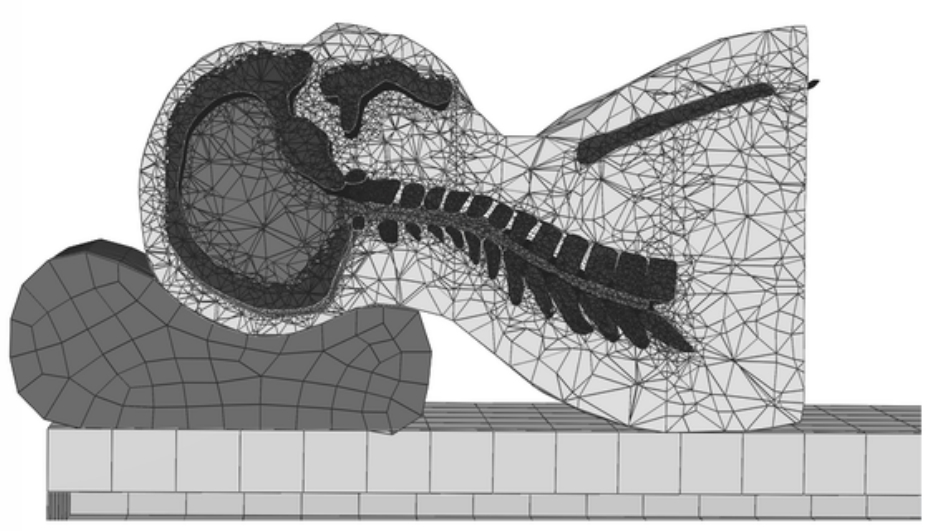




\section{Figure 3}

Illustration of the cervical spine alignment parameters

Figure 3. Illustration of the cervical spine alignment parameters: CA: cervical angle; LD:

lordosis distance; KD: kyphosis distance. The dashed line is a cubic spline regressed by the vertebrae coordinates. 


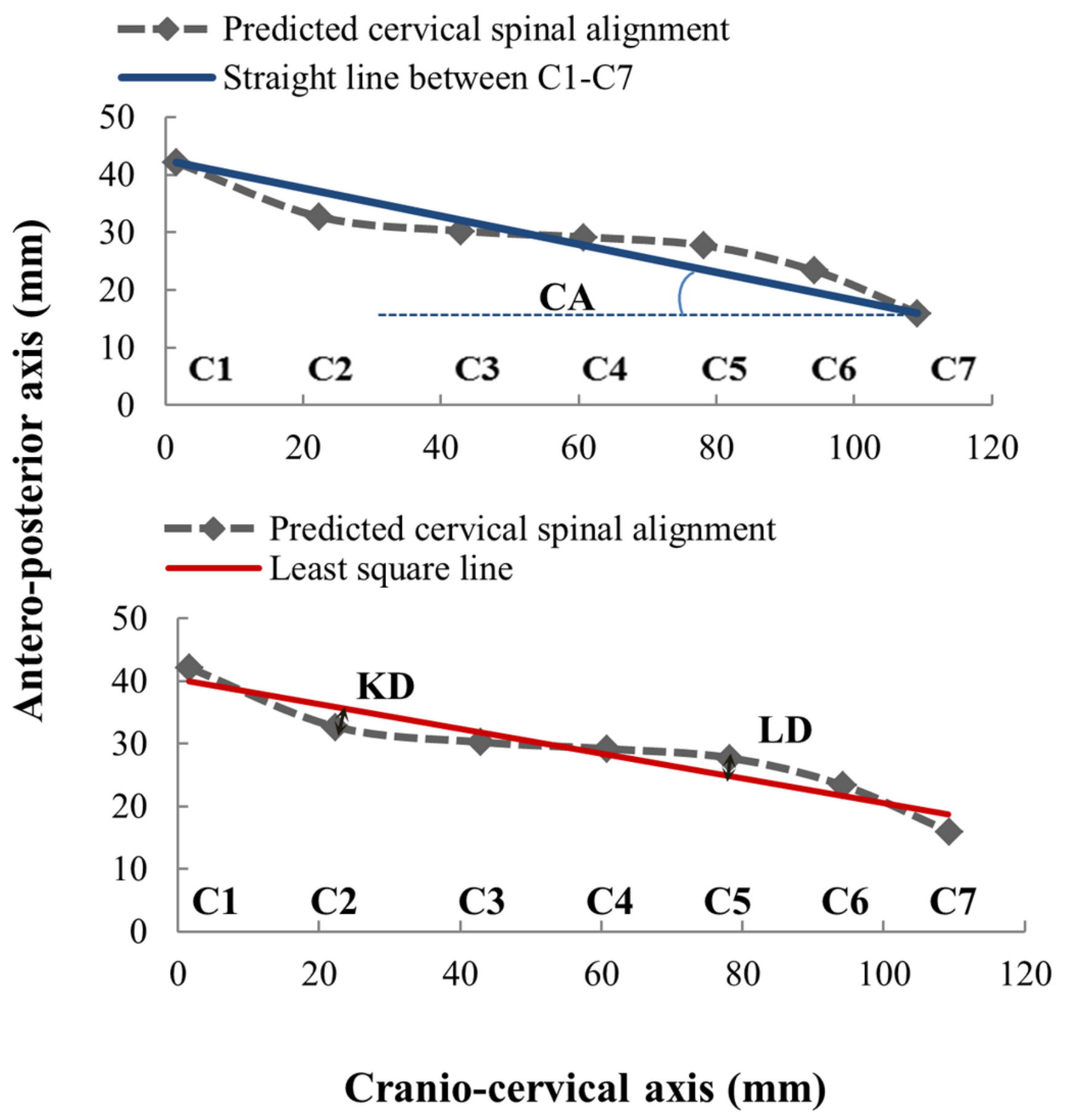




\section{Figure 4}

Cranio-cervical pressure distribution

Figure 4. Cranio-cervical pressure distribution under the four pillow height conditions from one typical subject.

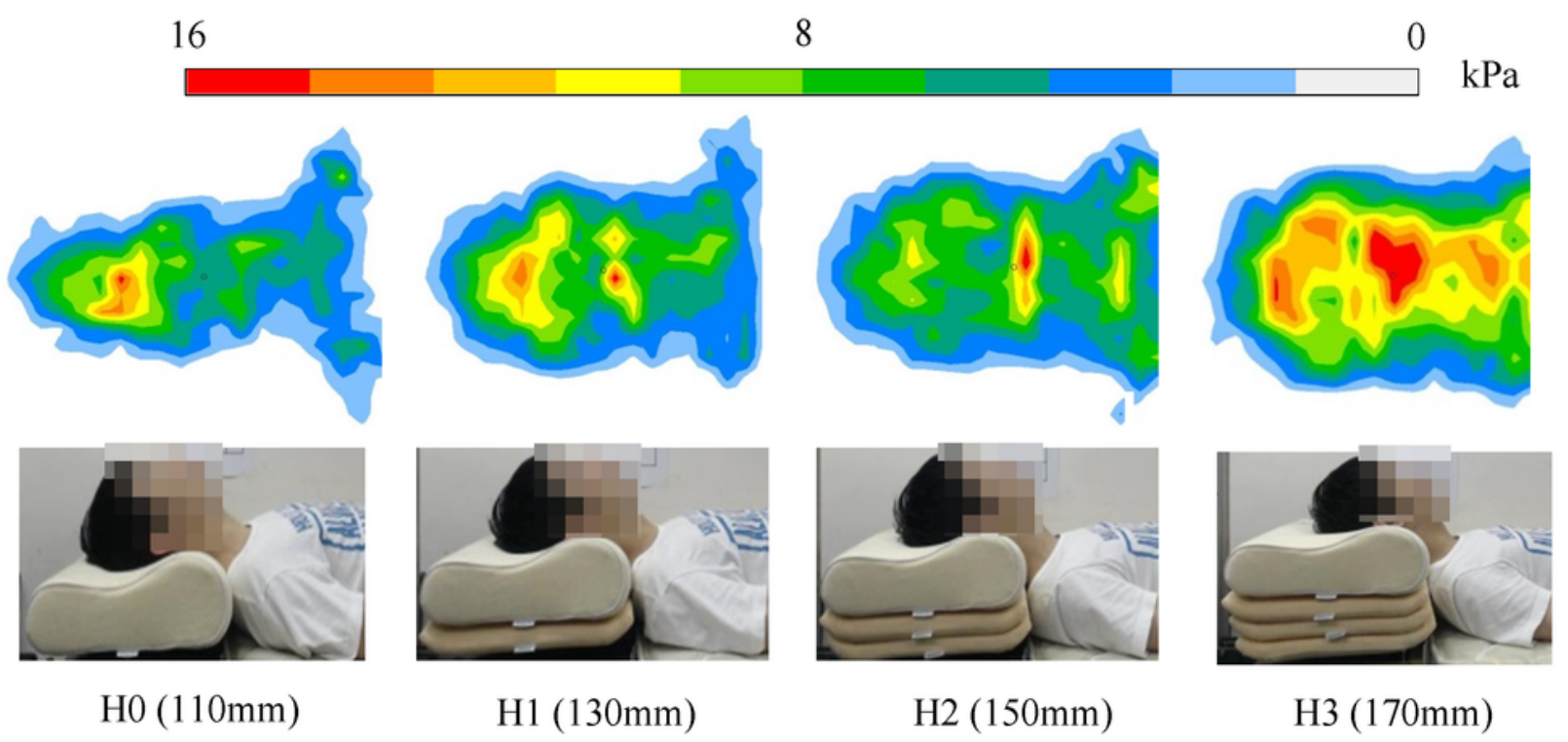


Figure 5

Finite element predicted position of the cervical vertebrae

Figure 5. Finite element predicted position of the cervical vertebrae under the four pillow height conditions.

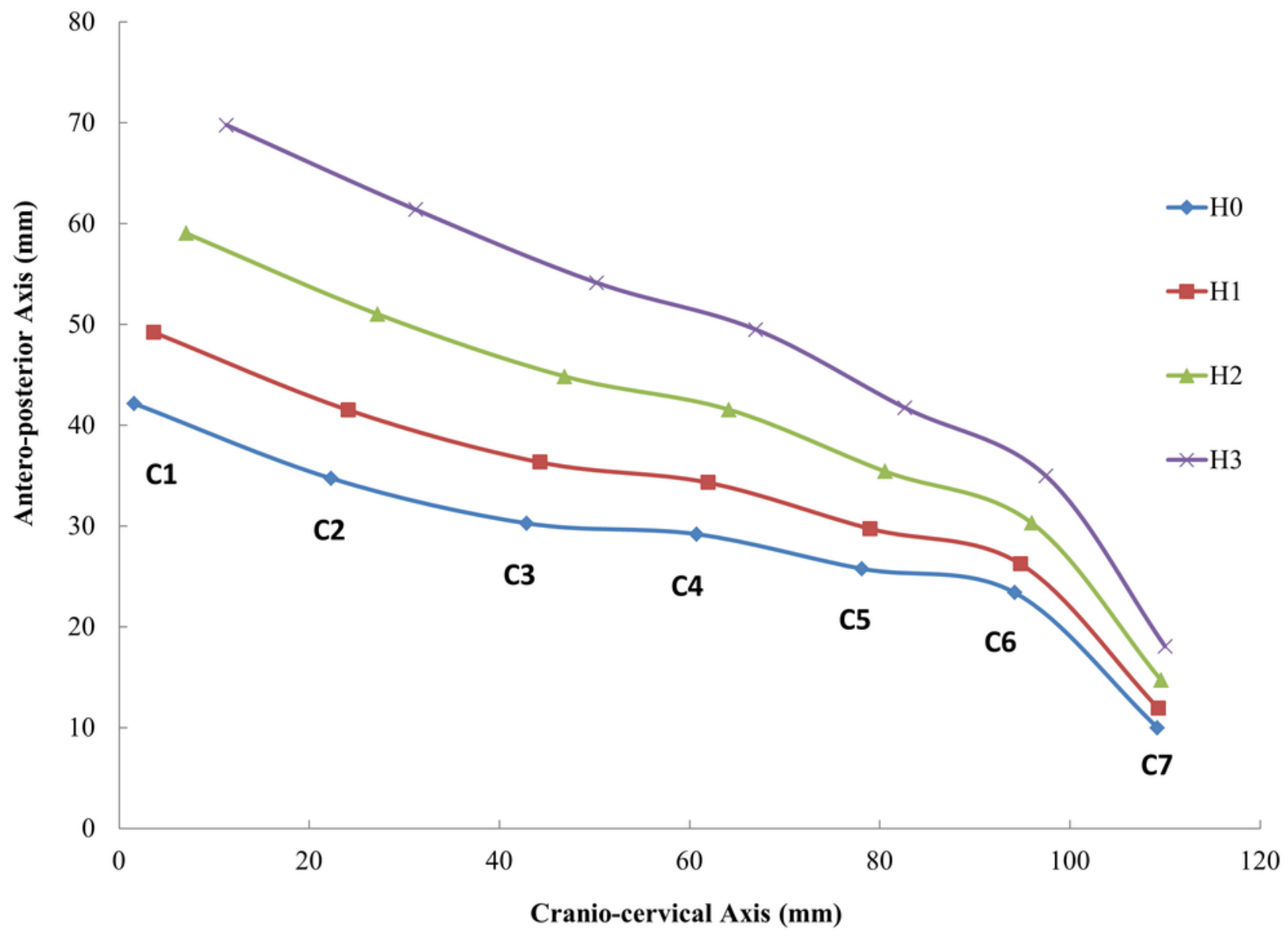




\section{Figure 6}

Comparison between physical experiment and finite element analysis

Figure 6. Comparison between physical experiment and finite element analysis under the four pillow height conditions: (a) peak cranio-cervical pressure; (b) cranial height.
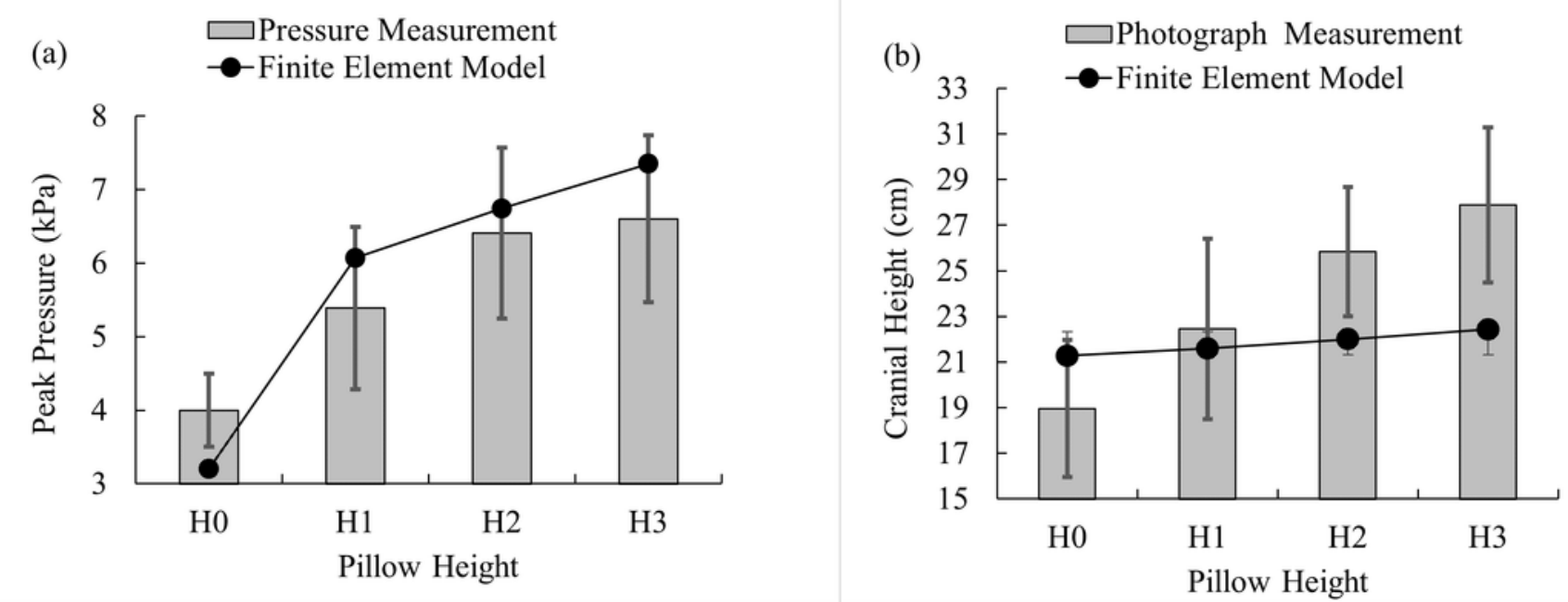


\section{Table $\mathbf{1}$ (on next page)}

Material properties of the finite element model 
1 Table 1. Material properties of the finite element model

\begin{tabular}{cccc}
\hline Component & Young's Modulus E (MPa) & Poisson's Ratio & Source \\
\hline Bone & 10000 & 0.3 & (Goel \& Clausen 1998) \\
Brain & 0.01 & 0.45 & (Soza et al. 2005) \\
Annulus fibrous & 450 & 0.45 & (Goel \& Clausen 1998) \\
Nucleus pulposus & 1 & 0.475 & (Mo et al. 2014) \\
Encapsulated Soft & Hyperelastic $\left(\mathrm{C}_{10}, \mathrm{C}_{01}, \mathrm{C}_{20}, \mathrm{C}_{11}, \mathrm{C}_{02}, \mathrm{D}_{1}, \mathrm{D}_{2}\right)=$ & (Lemmon et al. 1997; \\
Tissue & $(0.08556,-0.05841,0.03900,-0.02319,0.00851,3.65273,0)$ & Wang et al. 2015) \\
Pillow/Elevation mat & 0.054 & 0.045 & Measured by material \\
Bed & 2 & 0.3 & testing machine \\
\hline
\end{tabular}

2 
Table 2 (on next page)

Anthropometric data between male and female subjects 
1 Table 2. Anthropometric data between male and female subjects

\begin{tabular}{l|lll}
\multicolumn{1}{l}{} & Height (cm) & Weight $(\mathbf{k g})$ & Neck Length $(\mathbf{c m})$ \\
\hline Male & $178.4(4.39)$ & $68.1(11.02)$ & $14.19(1.26)$ \\
Female & $159.0(2.65)$ & $50.4(2.30)$ & $12.13(0.63)$ \\
Significance & $<0.01$ & $<0.01$ & $=0.01$
\end{tabular}

2 Significance level refers to independent sample t-test between male $(n=5)$ and female $(n=5)$ 


\section{Table 3 (on next page)}

Mixed ANOVA $(n=5)$ on gender and pillow height conditions demonstrating no significant interactions between the factors 
1 Table 3. Mixed ANOVA $(n=5)$ on gender and pillow height conditions demonstrating no significant 2 interactions between the factors

\begin{tabular}{|c|c|c|c|c|c|c|}
\hline \multirow{2}{*}{\multicolumn{2}{|c|}{ Cranial Region }} & HO & H1 & H2 & H3 & $\begin{array}{c}\text { Significance } \\
\text { of } \\
\text { Interaction }\end{array}$ \\
\hline & & & & & & \\
\hline Average & Male & $4.30(1.12)$ & $4.27(0.86)$ & $4.17(0.78)$ & $5.61(0.76)$ & \\
\hline Pressure & Female & $4.09(0.80)$ & $4.76(1.11)$ & $5.59(0.67)$ & $6.24(0.66)$ & 0.141 \\
\hline Peak & Male & $11.75(4.99)$ & $10.56(2.76)$ & $10.44(1.00)$ & $15.91(4.15)$ & \\
\hline Pressure & Female & $11.82(2.38)$ & $13.06(3.95)$ & $14.04(1.36)$ & $16.15(4.41)$ & 0.505 \\
\hline Cervical I & egion & & & & & \\
\hline Average & Male & $4.16(0.61)$ & $5.01(0.91)$ & $6.01(0.91)$ & $6.34(1.39)$ & \\
\hline Pressure & Female & $3.85(0.34)$ & $5.77(1.25)$ & $7.18(1.16)$ & $6.47(0.98)$ & 0.202 \\
\hline Peak & Male & $9.46(1.63)$ & $12.15(2.67)$ & $13.84(3.18)$ & $19.96(5.29)$ & \\
\hline Pressure & Female & $11.70(4.83)$ & $17.31(4.66)$ & $19.76(2.14)$ & $20.92(6.26)$ & 0.425 \\
\hline
\end{tabular}

3 Unit: $\mathrm{kPa}$ 


\section{Table 4 (on next page)}

Mean values $(n=10)$ of the average and peak cranial and cervical pressures under the four pillow height conditions 
1 Table 4. Mean values $(n=10)$ of the average and peak cranial and cervical pressures under

2 the four pillow height conditions

\begin{tabular}{ccccc}
\hline \multirow{2}{*}{ Pillow Height } & \multicolumn{4}{c}{ Mean Pressure (n=10) } \\
\cline { 2 - 5 } & \multicolumn{2}{c}{ Cranial Region (kPa) } & \multicolumn{2}{c}{ Cervical Region (kPa) } \\
\cline { 2 - 5 } & Average & Peak & Average & Peak \\
\hline H0 & $4.19(0.92)$ & $11.79(3.69)$ & $4.00(0.49)^{*}$ & $10.58(3.60)$ \\
H1 & $4.52(0.97)$ & $11.81(3.47)$ & $5.39(1.10)$ & $14.73(4.50)$ \\
H2 & $4.88(1.01)$ & $12.24(2.20)$ & $6.60(1.14)$ & $16.80(4.03)^{\#}$ \\
H3 & $5.93(0.75)^{*}$ & $16.03(4.03)$ & $6.41(1.16)$ & $20.44(5.49)^{\#}$ \\
\hline p & $<0.01$ & 0.02 & $<0.01$ & $<0.01$ \\
$\eta_{\text {partial }}^{2}$ & 0.504 & 0.338 & 0.693 & 0.568 \\
power & 0.583 & 0.359 & 0.961 & 0.690
\end{tabular}

3 Notes: Significance level refers to One-way ANOVA repeated measure on pillow height 4 conditions. * refers to significant difference compared to all other conditions in the post hoc 5 (Bonferroni) test. \# refers to significant difference compared to the baseline (H0) condition only 6 in the post hoc (Bonferroni) test. 


\section{Table 5 (on next page)}

Cervical spine alignment parameters in the four pillow height conditions predicted by finite element analysis 
1 Table 5. Cervical spine alignment parameters in the four pillow height conditions predicted

2 by finite element analysis

\begin{tabular}{cccc}
\hline Pillow Height & $\mathrm{CA}\left({ }^{\circ}\right)$ & $\mathrm{LD}(\mathrm{mm})$ & $\mathrm{KD}(\mathrm{mm})$ \\
\hline H0 & 16.62 & 5.41 & 1.96 \\
H1 & 19.44 & 5.71 & 1.75 \\
H2 & 23.36 & 6.18 & 1.44 \\
H3 & 27.65 & 6.77 & 1.11 \\
\hline
\end{tabular}

3 CA: Cervical Angle; LD: Lordotic Distance; KD: Kyphotic Distance 